\title{
Airway pressure release ventilation during ex vivo lung perfusion attenuates injury
}

\author{
J. Hunter Mehaffey, MD, ${ }^{\text {a }}$ Eric J. Charles, MD, ${ }^{a}$ Ashish K. Sharma, MBBS, PhD, \\ Dustin T. Money, RRT-ACCS, ${ }^{a}$ Yunge Zhao, MD, PhD, ${ }^{a}$ Mark H. Stoler, MD, ${ }^{b}$ \\ Christine L. Lau, MD, MBA, ${ }^{a}$ Curtis G. Tribble, MD, ${ }^{a}$ Victor E. Laubach, PhD, ${ }^{a}$ \\ Mark E. Roeser, MD, ${ }^{a}$ and Irving L. Kron, MD $^{a}$
}

\section{ABSTRACT}

Objective: Critical organ shortages have resulted in ex vivo lung perfusion gaining clinical acceptance for lung evaluation and rehabilitation to expand the use of donation after circulatory death organs for lung transplantation. We hypothesized that an innovative use of airway pressure release ventilation during ex vivo lung perfusion improves lung function after transplantation.

Methods: Two groups ( $\mathrm{n}=4$ animals/group) of porcine donation after circulatory death donor lungs were procured after hypoxic cardiac arrest and a 2-hour period of warm ischemia, followed by a 4-hour period of ex vivo lung perfusion rehabilitation with standard conventional volume-based ventilation or pressure-based airway pressure release ventilation. Left lungs were subsequently transplanted into recipient animals and reperfused for 4 hours. Blood gases for partial pressure of oxygen/inspired oxygen fraction ratios, airway pressures for calculation of compliance, and percent wet weight gain during ex vivo lung perfusion and reperfusion were measured.

Results: Airway pressure release ventilation during ex vivo lung perfusion significantly improved left lung oxygenation at 2 hours $(561.5 \pm 83.9 \mathrm{~mm} \mathrm{Hg}$ vs $341.1 \pm 136.1 \mathrm{~mm} \mathrm{Hg})$ and 4 hours $(569.1 \pm 18.3 \mathrm{~mm} \mathrm{Hg}$ vs $463.5 \pm 78.4 \mathrm{~mm}$ $\mathrm{Hg}$ ). Likewise, compliance was significantly higher at 2 hours $(26.0 \pm 5.2 \mathrm{~mL} / \mathrm{cm}$ $\mathrm{H}_{2} \mathrm{O}$ vs $\left.15.0 \pm 4.6 \mathrm{~mL} / \mathrm{cm} \mathrm{H}_{2} \mathrm{O}\right)$ and 4 hours $\left(30.6 \pm 1.3 \mathrm{~mL} / \mathrm{cm} \mathrm{H}_{2} \mathrm{O}\right.$ vs $17.7 \pm 5.9 \mathrm{~mL} / \mathrm{cm} \mathrm{H}_{2} \mathrm{O}$ ) after transplantation. Finally, airway pressure release ventilation significantly reduced lung edema development on ex vivo lung perfusion on the basis of percentage of weight gain $(36.9 \% \pm 14.6 \%$ vs $73.9 \% \pm 4.9 \%)$. There was no difference in additional edema accumulation 4 hours after reperfusion.

Conclusions: Pressure-directed airway pressure release ventilation strategy during ex vivo lung perfusion improves the rehabilitation of severely injured donation after circulatory death lungs. After transplant, these lungs demonstrate superior lung-specific oxygenation and dynamic compliance compared with lungs ventilated with standard conventional ventilation. This strategy, if implemented into clinical ex vivo lung perfusion protocols, could advance the field of donation after circulatory death lung rehabilitation to expand the lung donor pool. (J Thorac Cardiovasc Surg 2017;153:197-204)

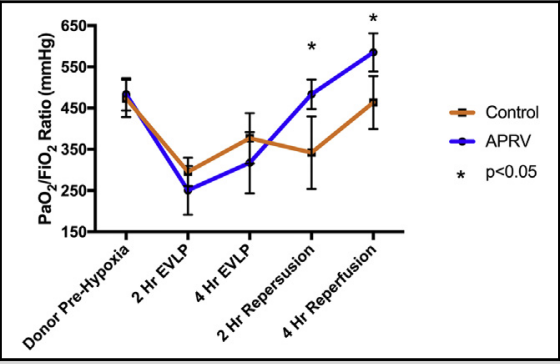

APRV during EVLP improves oxygenation posttransplant despite no difference in EVLP.

\section{Central Message}

Current EVLP ventilation strategies contribute to lung injury that can be attenuated with APRV.

\section{Perspective}

The use of EVLP for lung evaluation and rehabilitation before transplantation has been touted as a solution to the critical organ shortage. Volume-based ventilation using ARDSNet criteria leads to further lung injury during EVLP, which is attenuated with APRV The current study represents a paradigm shift in EVLP, necessitating reexamination of the current clinical practice.

See Editorial Commentary page 205.
Although the yearly number of lung transplants has increased more than 35 -fold in the past 20 years and continues to increase, the number of organ donors has remained

From the Departments of ${ }^{\mathrm{a}}$ Surgery and ${ }^{\mathrm{b}}$ Pathology, University of Virginia, Charlottesville, Va.

The National Heart, Lung, and Blood Institute of the National Institutes of Health under Award Numbers T32HL007849 and R01HL119218 supported the research reported in this publication. The content is solely the responsibility of the authors and does not necessarily represent the official views of the National Institutes of Health. Read at the 42nd Annual Meeting of The Western Thoracic Surgical Association, Waikoloa, Hawaii, June 24, 2016. mostly static. ${ }^{1}$ This donor shortage is exacerbated by the fact that, in general, only $15 \%$ of lungs from multiorgan donors are deemed suitable for transplantation. Thus, many

Received for publication June 20, 2016; revisions received Aug 13, 2016; accepted for publication Sept 13, 2016; available ahead of print Oct 11, 2016.

Address for reprints: Irving L. Kron, MD, University of Virginia, PO Box 800679, Charlottesville, VA 22908 (E-mail: ilk@virginia.edu). 0022-5223/\$36.00

Copyright (C) 2016 by The American Association for Thoracic Surgery http://dx.doi.org/10.1016/j.jtcvs.2016.09.029 


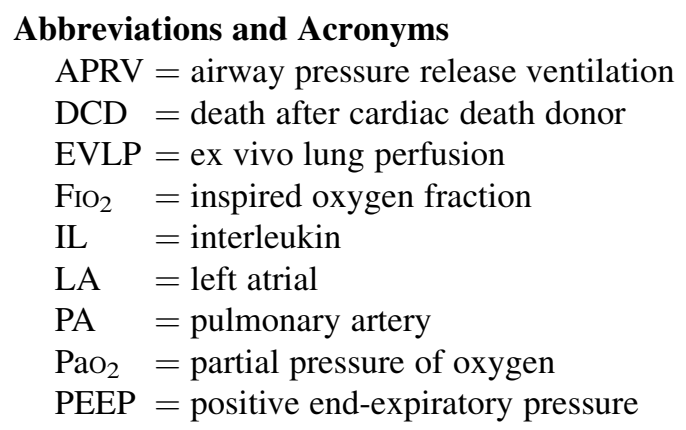

Scanning this QR code will take you to a video for the article.

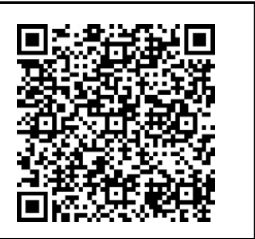

patients die while waiting for a suitable donor, with wait-list mortality as high as $30 \%$ to $40 \%{ }^{2}$ Despite all current strategies implemented to increase the availability of donor lungs, the majority of potential lungs are still rejected for transplantation.

Recently, a pioneering strategy using a "lung box" for ex vivo lung perfusion (EVLP) has demonstrated significant potential to address both the quantity and the quality of available organs. ${ }^{3}$ The EVLP system was originally described by Steen and colleagues ${ }^{4}$ and further modified by the Toronto group to the current ex vivo protocol using Steen solution..$^{5}$ This innovative method maintains the lungs in physiologically protective conditions outside the body during preservation and allows accurate evaluation of lung function, as well as providing a new setting for therapeutic treatment and repair of damaged donor lungs before transplantation.

Stock and colleagues ${ }^{6}$ first described airway pressure release ventilation (APRV) in the 1980s. APRV is a pressure-directed mode of ventilation providing 2 levels of positive airway pressure, with the majority of time spent at the high level and a brief expiratory release at the lower pressure to facilitate ventilation. ${ }^{7}$ This mode has many reported advantages over conventional ventilation, including alveolar recruitment, improved oxygenation, improved hemodynamics, and attenuation of barotrauma. ${ }^{8-10}$ Many of these advantages can be translated into the EVLP model to improve atelectatic lung recruitment, reduce pulmonary edema, and ameliorate barotrauma.

As EVLP gains popularity, it is crucial to examine the ventilation protocol used in this system. A recent article by Terragni and colleagues ${ }^{11}$ demonstrated that the current EVLP ventilator settings may expose the lungs to ventilator-induced lung injury. The original volume-based approach was based on the ARDSNet criteria for in vivo lungs confined by the chest wall. ${ }^{3}$ The current study examines the use of APRV during EVLP to reduce barotrauma and prevent further lung injury. We hypothesized that this innovative use of APRV will improve atelectatic lung recruitment, reduce pulmonary edema, and attenuate barotrauma after lung transplantation.

\section{MATERIALS AND METHODS \\ Animals and Study Groups}

The current study complies with the 1996 Guide for the Care and Use of Laboratory Animals as recommended by the US National Institutes of Health and was approved by the University of Virginia Animal Care and Use Committee. All animals received humane care during the duration of the study. Adolescent domestic swine of both sexes (24-39 kg) underwent hypoxic cardiac arrest. After 2 hours of no-touch warm ischemia, cold preservation flush with Perfadex (XVIVO Perfusion Inc, Englewood, Colo) was performed and the lungs were procured. The experimental group $(n=4)$ received APRV during EVLP, which was compared with a group of historical controls $(n=4)$ receiving conventional ventilation during EVLP.

\section{Lung Injury and Procurement}

After the 2-hour warm ischemia period, procurement of donation after cardiac death lungs was completed as previously described. ${ }^{12}$ Animals were sedated and weighed, followed by induction of anesthesia and intubation. Donor animals were ventilated with $100 \%$ oxygen during the measurement of baseline values. Before crossclamping the endotracheal tube to induce hypoxic cardiac arrest, donor animals received intravenous heparin $(200 \mathrm{U} / \mathrm{kg}$; Hospira Inc, Lake Forest, Ill), and initial donor $\mathrm{PaO}_{2} / \mathrm{FiO}_{2}$ ratio was obtained from a right carotid arterial blood gas sample. After crossclamp, it took a median of 18 minutes for the animals to die, and there was typically some respiratory effort during this period. Death was confirmed with continuous electrocardiogram monitoring; the animal underwent 2 hours of no-touch warm ischemia. During the final 5 minutes of the warm ischemia period, ventilation was resumed and the donor underwent standard cold preservation flush with Perfadex and bilateral lung procurement (Video 1).

A median sternotomy was performed, and a cardioplegia cannula (Terumo Heart Inc, Ann Arbor, Mich) was placed into the main pulmonary

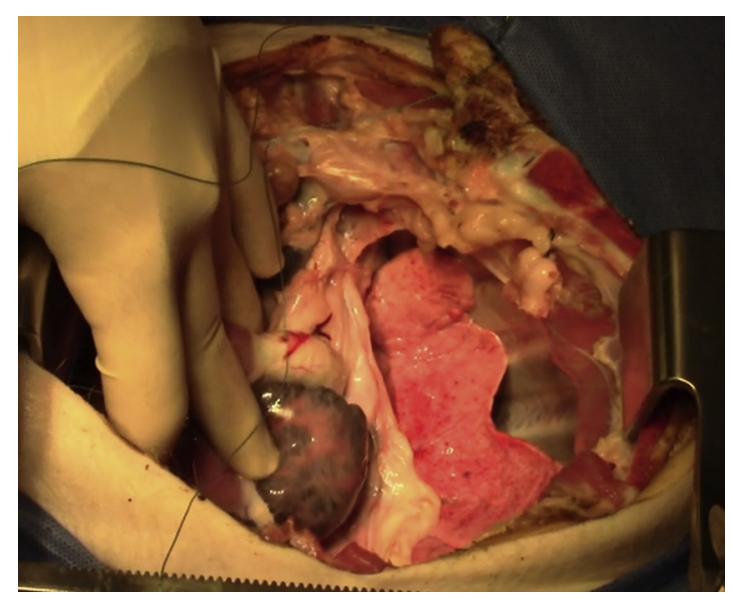

VIDEO 1. Porcine procurement and left lung transplant model. Video available at: http://www.jtcvsonline.org/article/S0022-5223(16)31147-3/ addons. 
artery (PA) for delivery of prostaglandin-E1 (500 $\mu \mathrm{g}$, Pfizer Inc, New York, NY) followed by a cold Perfadex flush. The initial flush was 1.5 liters of Perfadex supplemented with $15,000 \mathrm{IU}$ of heparin after ligating the superior and inferior vena cavae and venting the left atrial (LA) appendage. The trachea was crossclamped mid-inspiration to maintain partial inflation of the lungs, and the heart-lung bloc was explanted from the donor animal.

\section{Ex Vivo Lung Perfusion}

After back-table preparation, a yellow cannula (XVIVO Perfusion Inc) was tied in the main PA, a green cannula (XVIVO Perfusion Inc) was sewn to the LA cuff, and a 7-0 endotracheal tube was tied into the trachea. Before initiation of 4 hours of EVLP, an additional $500 \mathrm{~mL}$ of cold Perfadex was flushed retrograde through the LA cannula. The lung block with cannulas was then weighed before initiation of EVLP for baseline weight.

EVLP was initiated on a perfusion circuit as previously described.$^{13}$ The circuit was primed in the standard fashion with Steen Solution, cefazolin (500 mg, APP Pharmaceuticals, Schaumburg, Ill), methylprednisolone (500 mg, Pfizer Inc), and heparin (10,000 IU). The circuit was perfused according to the protocol described by the Toronto group with flow initiated $(0.2 \mathrm{~mL} / \mathrm{min})$ and LA pressures maintained between 0 and $5 \mathrm{~mm} \mathrm{Hg}{ }^{3}$ The perfusate was gradually warmed to $37^{\circ}$ over the first 30 minutes as flow was titrated up to $40 \%$ of estimated cardiac output $(100 \mathrm{~mL} / \mathrm{kg}$ donor body weight). A standard tri-gas mixture ( $86 \%$ nitrogen, $8 \%$ carbon dioxide, $6 \%$ oxygen) through an Affinity membrane (Medtronic Inc, Eden Prairie, Minn) was used to deoxygenate the perfusate.

When perfusate temperature reached $35^{\circ} \mathrm{C}$, ventilation was initiated with conventional ventilation (tidal volume $8 \mathrm{~mL} / \mathrm{kg}$, respiratory rate 8 breaths/min, positive end-expiratory pressure [PEEP] $5.0 \mathrm{~cm} \mathrm{H}_{2} \mathrm{O}$, inspired oxygen fraction $\left[\mathrm{FIO}_{2}\right] 0.21$ ) or APRV ( $\mathrm{T}_{\text {high }} 30.0$ seconds, $\mathrm{T}_{\text {Low }} 1.0$ second, $\mathrm{P}_{\text {High }} 10.0 \mathrm{~cm} \mathrm{H}_{2} \mathrm{O}, \mathrm{P}_{\text {Low }} 0.0 \mathrm{~cm} \mathrm{H} \mathrm{H}_{2} \mathrm{O}$ ). $\mathrm{P}_{\text {High }}$ was titrated to target tidal volume $6 \mathrm{~mL} / \mathrm{kg}$ with max $\mathrm{P}_{\mathrm{High}}$ of $10 \mathrm{~cm} \mathrm{H} \mathrm{H}_{2} \mathrm{O}$ for the first hour, $15 \mathrm{~cm} \mathrm{H}_{2} \mathrm{O}$ for the second hour, and never more than $20 \mathrm{~cm} \mathrm{H}_{2} \mathrm{O}$ for the last 2 hours. We opted to use the longer $\mathrm{T}_{\text {Low }}$ with a slightly higher $\mathrm{P}_{\mathrm{High}}$ to prevent auto-PEEP, which could be a major issue in open lung ventilation without a chest wall. Perfusate samples from the PA inflow and LA outflow were collected every hour after a 15-minute challenge period with $1.0 \mathrm{FIO}_{2}$ to measure the partial pressure of oxygen $\left(\mathrm{PaO}_{2}\right)$. Airway pressures on conventional ventilation were measured hourly to calculate dynamic compliance.

After 4 hours of EVLP, the lungs were removed from the circuit and weighed for calculation of edema gain on EVLP. Subsequently, the lung block was flushed anterograde with $500 \mathrm{~mL}$ of cold Perfadex. The left lung was then split and prepared on the back-table for subsequent transplantation, and the right lung was taken for fresh tissue samples and histology. Just before transplantation, the left lung was weighed again for baseline weight before reperfusion.

\section{Left Lung Transplant and Reperfusion}

Recipient animals were anesthetized and ventilated with conventional ventilation in the same manner as the donor animals. After induction of anesthesia, a central line was placed in the internal jugular vein with a Swan-Ganz catheter and an arterial line in the carotid. The animals were all maintained on conventional ventilation (tidal volume $8 \mathrm{~mL} / \mathrm{kg}$, respiratory rate $12-16$ breaths/min, PEEP $5 \mathrm{~mm} \mathrm{Hg}$ ) with isoflurane $(3 \%)$ and $100 \%$ oxygen. A left lateral thoracotomy was then performed, and the animal received $5000 \mathrm{IU}$ of heparin just before left pneumonectomy and left lung transplant (running sutures used for end-to-end bronchial anastomosis, end-to-end PA anastomosis, and LA cuff to recipient LA appendage) as described previously. ${ }^{13}$ The median procedure time was 42 minutes (interquartile range, 38-44) (Video 1).

Post-transplant reperfusion of the left donor lung was maintained for 4 hours with conventional ventilation (tidal volume $8 \mathrm{~mL} / \mathrm{kg}$, respiratory rate $12-16$ breaths/min, PEEP $5 \mathrm{~mm} \mathrm{Hg}$ ) and $100 \%$ oxygen. The left thoracotomy was left wide open with suspension from above to prevent chest wall
TABLE 1. Summary of grading criteria for the Lung Injury Severity Score

\begin{tabular}{lccc}
\hline Score & PMNs per HPF & Alveolar edema & Interstitial inflammation \\
\hline 0 & $<5$ & $<5 \%$ & None \\
1 & $6-10$ & $6-25 \%$ & Minimal \\
2 & $11-20$ & $26-50 \%$ & Moderate \\
3 & $>20$ & $>50 \%$ & Severe \\
\hline
\end{tabular}

Maximum score is 9 , and minimum score is $0 . P M N$, Polymorphonuclear leukocytes; $H P F$, high-powered field.

restriction on the lung. Airway pressure measurements and carotid arterial blood gases were performed every hour during reperfusion. Superior and inferior pulmonary vein blood gas samples were obtained at 2 hours and 4 hours of reperfusion for left lung-specific $\mathrm{PaO}_{2} / \mathrm{FIO}_{2}$ ratio. Hemodynamic goals were $\mathrm{pH} 7.35$ to 7.45 , base excess greater than -5 , and mean arterial pressure greater than $55 \mathrm{~mm} \mathrm{Hg}$, which were maintained with the use of normal saline, epinephrine, and sodium bicarbonate as necessary. The donor lung was explanted after 4 hours of reperfusion and weighted for measurement of edema gain during reperfusion. The animal was then euthanized.

\section{Cytokine Measurements}

After 4 hours of EVLP, 3 fresh tissue samples were obtained (upper lobe, upper portion of the lower lobe, and lower portion of the lower lobe) from the right lung of the APRV group to establish a baseline for inflammatory markers. After 4 hours of post-transplant reperfusion, 3 tissue samples were taken from the transplanted lung (left) of both the APRV and control groups. The fresh tissue was flash-frozen in liquid nitrogen and stored at $-80^{\circ} \mathrm{C}$. After homogenization with a FastPrep-24 (MP Biomedicals, Santa Ana, Calif), the total protein concentration in the supernatant of each homogenized lung tissue sample was determined with a bicinchoninic acid protein assay (Pierce, Rockford, Ill). Multiplex enzyme-linked immunosorbent assay (EMD Millipore, Billerica, Mass) was used to measure the cytokine levels in the tissue supernatant (normalized to equal protein concentrations).

\section{Histology}

After obtaining fresh tissue samples, the airways of the lower lobe were filled with $10 \%$ buffered formalin. After overnight fixation in formalin, peripheral lung tissue samples $(n=4 / \mathrm{lung}$ ) were obtained, paraffinembedded, and sectioned. One slide from each sample was stained with hematoxylin-eosin, and 2 slides (1 from the upper most sample and 1 from the lower most sample) were used for immunohistochemistry evaluation of activated neutrophil infiltration.

A masked pathologist assessed the hematoxylin-eosin-stained slides for the presence of lung injury. Each slide was scored on a standard scale based on polymorphonuclear cells per $40 \times$ high-powered field, alveolar edema, and interstitial inflammation as previously described. ${ }^{14}$ The grading scale is listed in Table 1.

For neutrophil immunohistochemistry staining, mouse monoclonal antiporcine neutrophil antibody (MBA Biomedicals, Augst, Switzerland) was the primary antibody, and donkey antimouse immunoglobulin-G (Jackson ImmunoResearch Laboratories Inc, West Grove, Pa) was the secondary antibody. Standard protocol using an avidin-biotin complex followed by incubation with 3,3-diaminobenzidine tetrahydrochloride (Dako Inc, Carpinteria, Calif) to produce a brown precipitate and hematoxylin counterstain was used as previously described. ${ }^{15}$ Microscopic photographs were taken at $40 \times$ magnification of each slide, and the number of neutrophils per high-powered field were counted by a blinded investigator.

\section{Statistical Analysis}

Student $t$ test and Fisher exact test were used to determine statistical significance. Prism 7 (GraphPad Software Inc, La Jolla, Calif) was used to 


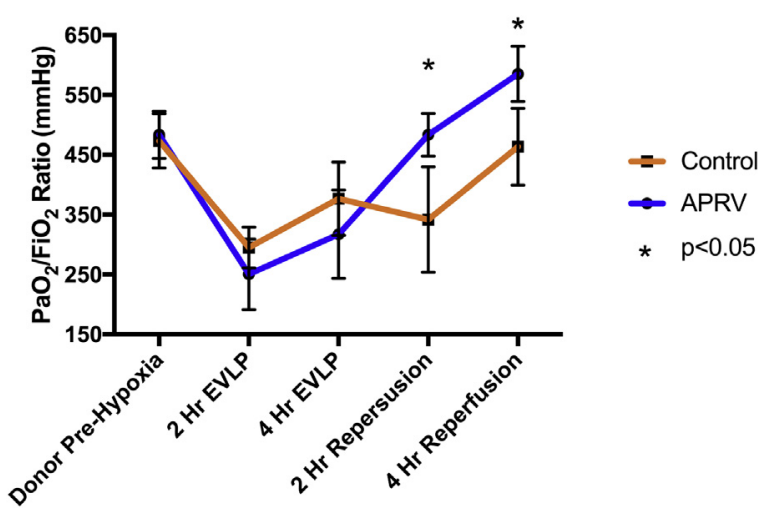

FIGURE 1. Changes in $\mathrm{PaO}_{2} / \mathrm{FIO}_{2}$ ratios of the left lung over the course of the experiment. $\mathrm{PaO}_{2} / \mathrm{FIO}_{2}$ ratios were significantly higher in the APRV group after transplantation at both the 2-hour and 4-hour reperfusion times. $\mathrm{PaO}_{2}$, Partial pressure of oxygen; $\mathrm{FIO}_{2}$, inspired oxygen fraction; $\mathrm{APRV}$, airway pressure release ventilation; $E V L P$, ex vivo lung perfusion.

perform statistical calculations, and all data are reported as mean \pm standard deviation.

\section{RESULTS}

\section{Lung Function and Edema}

Lung-specific oxygenation over the course of the experiment is illustrated in Figure 1 for each group. There was no difference in prehypoxia donor $\mathrm{PaO}_{2} / \mathrm{FIO}_{2}$ ratios (483.4 $\pm 39.4 \mathrm{~mm} \mathrm{Hg}$ vs $473.2 \pm 45.2 \mathrm{~mm} \mathrm{Hg}, P>.05)$. In addition, during EVLP there was no difference in oxygenation at 2 or 4 hours between lungs on APRV versus those on conventional ventilation $(250.5 \pm 59.0 \mathrm{~mm} \mathrm{Hg}$ vs $295.2 \pm 34.1 \mathrm{~mm} \mathrm{Hg}$ at 2 hours, $317.5 \pm 74.0 \mathrm{~mm} \mathrm{Hg}$ vs $376.7 \pm 61 \mathrm{~mm} \mathrm{Hg}$ at 4 hours, all $P>.05$ ). However, after transplant and reperfusion, a significant improvement in oxygenation at both 2 and 4 hours was observed in the group receiving APRV during EVLP $(483.4 \pm 35.7 \mathrm{~mm} \mathrm{Hg}$ vs $341.9 \pm 87.9 \mathrm{~mm} \mathrm{Hg}$ at 2 hours, $585.1 \pm 46.2 \mathrm{~mm} \mathrm{Hg}$ vs $463.5 \pm 64.0 \mathrm{~mm} \mathrm{Hg}$ at 4 hours, all $P<.05$ ).

Despite starting at a higher $\mathrm{PaO}_{2} / \mathrm{FiO}_{2}$ ratio in the control group $(375.0 \pm 23.3 \mathrm{~mm} \mathrm{Hg}$ vs $201.0 \pm 60.2 \mathrm{~mm} \mathrm{Hg}$, $P=.02$ ), after 1 hour of EVLP there was no difference in oxygenation during hours 2 to 4 of EVLP between the groups (all $P>.05$ ) (Figure 2, A). Oxygenation improved over the 4 hours of EVLP in both groups. Likewise, there was no difference (all $P>.05$ ) in dynamic compliance during the 4 hours of EVLP, with both groups demonstrating a slight improvement (Figure 2, B).

After left lung transplant, the left pulmonary vein gases in the group that received APRV during EVLP were superior at both 2 and 4 hours versus the group that received conventional ventilation on EVLP (Figure 3,A). Dynamic compliance again was significantly improved at 2 and 4 hours of reperfusion in the group receiving APRV during EVLP (Figure 3, $B$ ).

Figure 3, C demonstrates significantly less percent weight gain secondary to pulmonary edema during EVLP in the APRV group compared with the conventional ventilation group $(36.9 \% \pm 12.7 \%$ vs $73.9 \% \pm 4.2 \%$, $P=.03$ ). However, there was no statistical difference in weight gain during reperfusion between the 2 groups $(14.5 \% \pm 6.5 \%$ vs $32.8 \% \pm 22.7 \%, P=.22)$.

\section{Cytokine Expression}

Biologically relevant proinflammatory cytokines were measured in lung tissue samples from right lung tissue after EVLP in group APRV and left lung tissue after reperfusion in the control group and APRV group (Figure 4). There was a higher expression of interleukin (IL)-6, IL- $1 \beta$, and IL-4 in the postreperfusion tissues of both control and APRV versus the post-EVLP right lung tissue. In addition, IL-6 expression after transplantation was significantly reduced in the APRV group versus control $(P=.04)$. IL-12 expression, which was elevated after transplantation in the control group, was significantly reduced in the APRV group $(P=.04)$, similar to the IL-12 levels in the post-EVLP lungs.

\section{Lung Injury Severity Score and Neutrophil Infiltration}

Although the lung injury severity scores from 4 matched samples in each lung were lower after transplantation in the APRV group $(4.5 \pm 1.3$ vs $5.9 \pm 0.9, P=.11)$, there was no
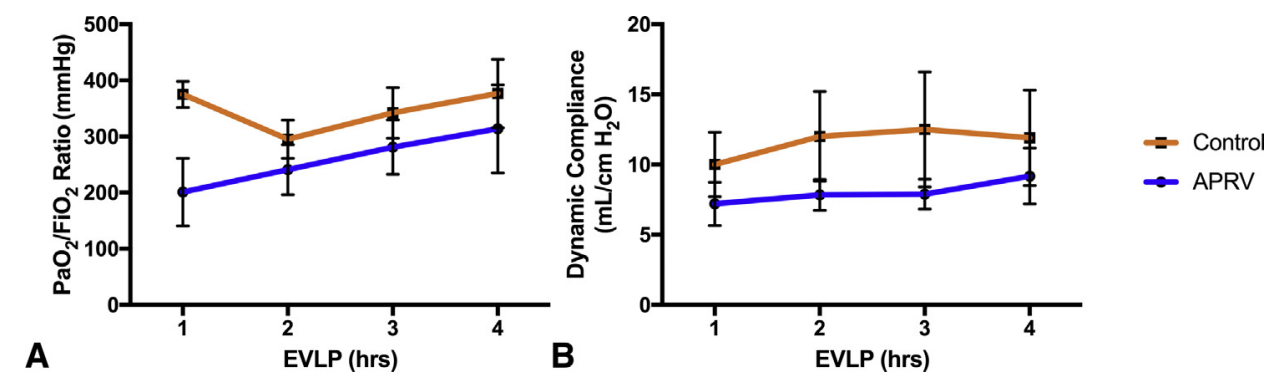

FIGURE 2. Changes in $\mathrm{PaO}_{2} / \mathrm{FIO}_{2}$ ratios and dynamic compliance during EVLP. A, No significant differences in $\mathrm{PaO}_{2} / \mathrm{FIO}_{2}$ ratios during EVLP were observed between the control and APRV groups. B, Dynamic compliance throughout the 4 hours of EVLP was similar for both groups. Pao ${ }_{2}$, Partial pressure of oxygen; $\mathrm{FIO}_{2}$, inspired oxygen fraction; $A P R V$, airway pressure release ventilation; $E V L P$, ex vivo lung perfusion. 

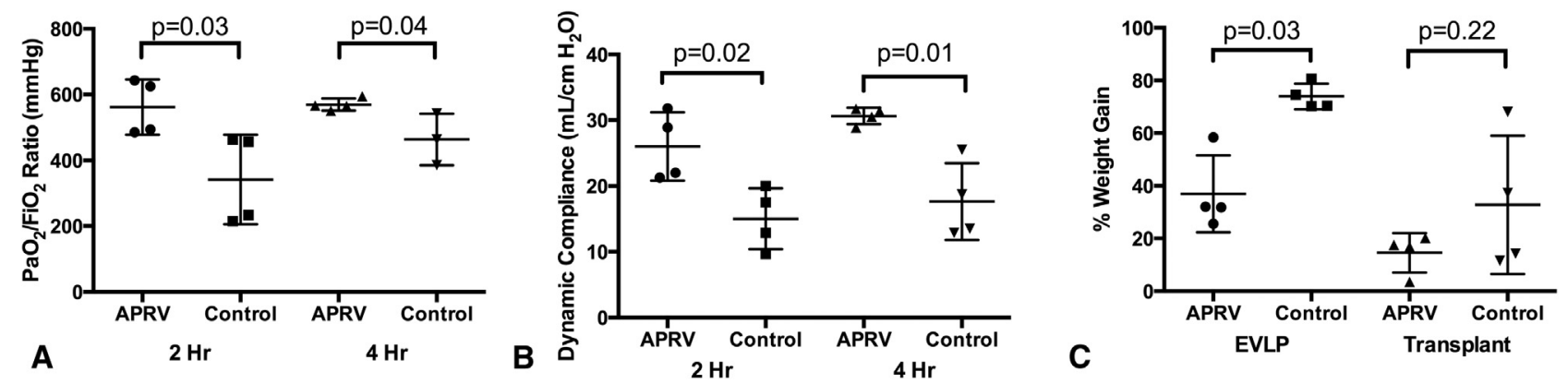

FIGURE 3. Lung function and edema after transplantation. $\mathrm{PaO}_{2} / \mathrm{FIO}_{2}$ ratios $(\mathrm{A})$ and dynamic lung compliance (B) were significantly improved in the APRV group at both 2 and 4 hours of post-transplant reperfusion. C, Pulmonary edema, as a percent weight change after 4 hours of reperfusion, was significantly reduced in the APRV group during EVLP versus control, but there was no significant difference after post-transplant reperfusion. $\mathrm{PaO}_{2}$, Partial pressure of oxygen; $\mathrm{FIO}_{2}$, inspired oxygen fraction; $\mathrm{APRV}$, airway pressure release ventilation; EVLP, ex vivo lung perfusion.

statistical difference between the groups (Figure 5). In addition, on immunohistochemistry staining for neutrophils of 2 matched samples per lung, the APRV group had significantly less neutrophil infiltration (fewer neutrophils per high-powered field: $74.2 \pm 29.8$ vs $111.7 \pm 10.0$, $P=.01$ ) versus the control group (Figure 6).

\section{DISCUSSION}

By using a clinically relevant porcine model of EVLP and left lung transplant after death after cardiac death donor (DCD) procurement, we demonstrated beneficial effects of APRV during EVLP on post-transplant lung performance. Lung-specific oxygenation, dynamic compliance, and pulmonary edema represent the most important clinical markers of early transplant function, which were all dramatically improved in the APRV group. In addition, the biochemical markers of inflammation, including IL-6 and IL-12, were significantly lower in the APRV group and closer to the baseline levels observed in post-EVLP lungs before reperfusion. Histologic assessment suggests lower injury severity scores and reduced neutrophil infiltration in the APRV group compared with the controls.

$\mathrm{PaO}_{2} / \mathrm{FIO}_{2}$ ratios as a measure of lung oxygenation capacity traditionally have been used as the most important
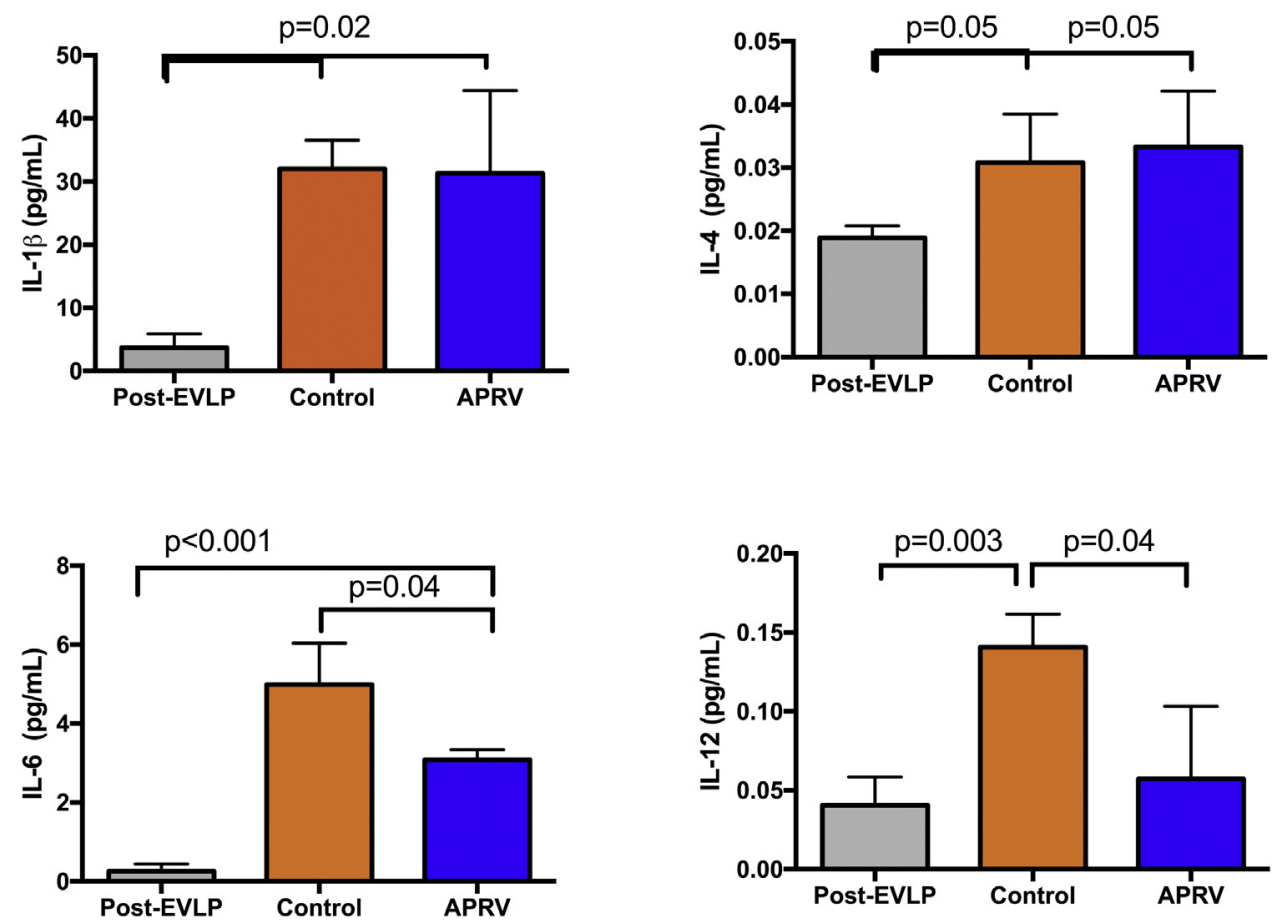

FIGURE 4. Lung tissue expression of proinflammatory cytokines in the left lungs of the APRV and control groups, as well as in post-EVLP right lungs. Several cytokines (IL-6 and IL-12) were significantly elevated in the control group but significantly reduced in the APRV group. IL, Interleukin; $E V L P$, ex vivo lung perfusion; $A P R V$, airway pressure release ventilation. 

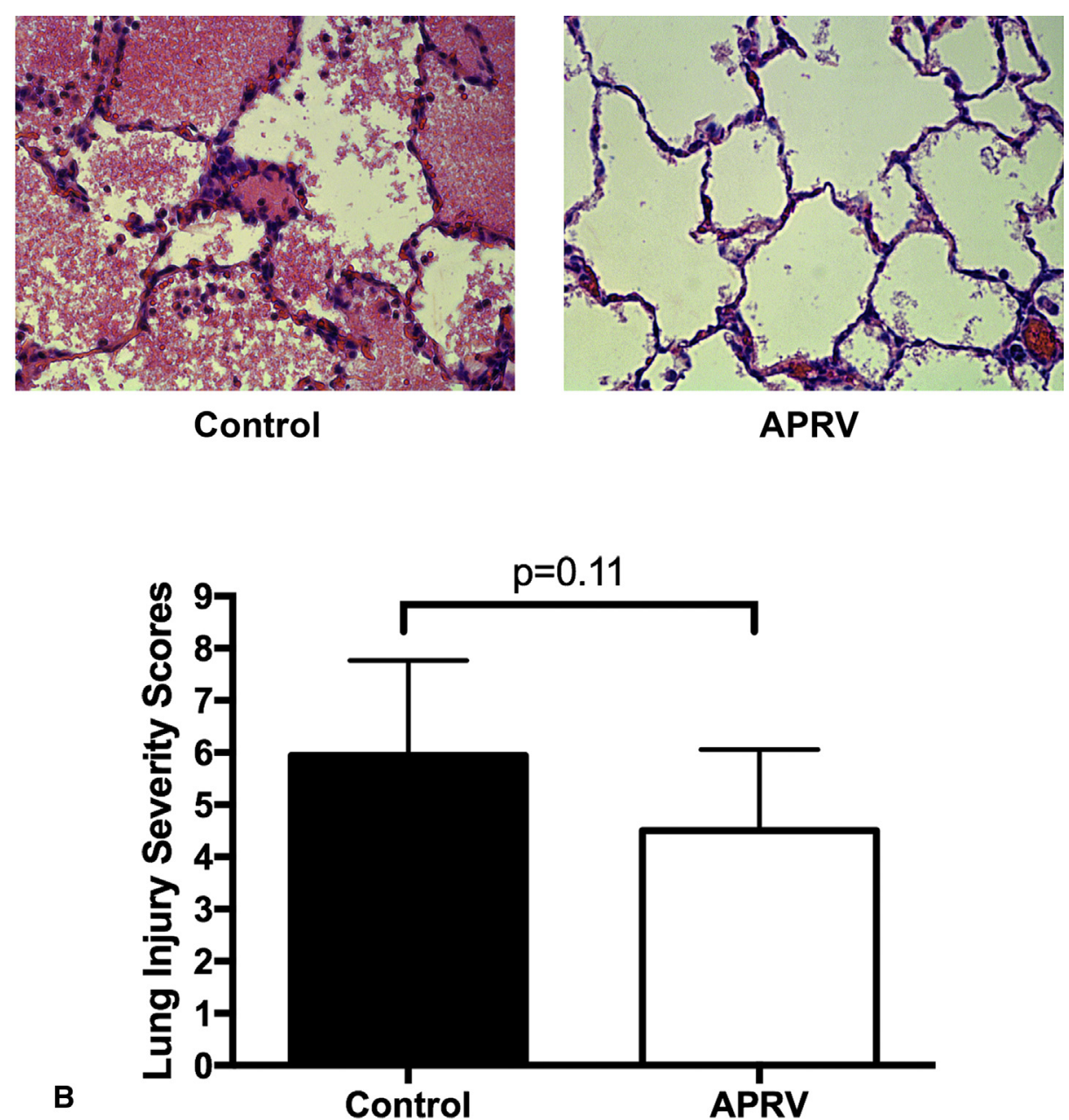

FIGURE 5. Representative lung hematoxylin-eosin histology images from the control and APRV groups (A). Although lower in the APRV group, lung injury severity scores were not significantly different between the control and APRV groups (B). APRV, Airway pressure release ventilation.

predictor of successful lung transplantation; however, our group and others have demonstrated the importance of lung compliance. ${ }^{3,16}$ In the present study, both of these markers of lung function improved after reperfusion in the group receiving APRV during EVLP. The reduction in pulmonary edema during EVLP is likely the main cause of this difference. By keeping the alveoli continuously recruited with APRV during EVLP, the surface tension reduces fluid translocation. ${ }^{10,17}$ It is important to note that even after transplant and reperfusion with conventional ventilation, lungs undergoing APRV on EVLP did not have additional edema during the reperfusion period. These clinical parameters demonstrate that APRV recruitment on EVLP has post-transplant benefits and should be considered for translation into human studies.

The biochemical profile of these lungs after EVLP and subsequent transplant represents a complex interaction between donor lung ischemia-reperfusion injury with the initiation of EVLP and subsequent recipient cell activation and injury after transplant and reperfusion. IL-6 is part of the tumor necrosis factor- $\alpha$-activated proinflammatory pathway that has been noted by several groups, including our own, to be a component of ischemia-reperfusion injury after lung transplant. ${ }^{16,18-22}$ However, Farivar and colleagues $^{23}$ demonstrated reduced endothelial disruption and neutrophil sequestration with recombinant IL-6 in a rat ischemia-reperfusion model of lung transplant highlighting the signal orchestration that can lead to both proinflammatory and anti-inflammatory effects of this cytokine. ${ }^{23}$ In the present study, IL-6 levels are significantly higher in the transplanted lungs compared with post-EVLP lungs but lower in the APRV-treated group versus the control group after reperfusion. Although the tissue level expression of IL-6 is correlated with increased neutrophil counts, EVLP may wash out the intermediary tumor necrosis factor- $\alpha$ signal. IL-12 has been cited as a proinflammatory cytokine in the inflammosome pathway. ${ }^{24,25}$ Our group previously demonstrated a benefit from IL-12 deregulation through the adenosine 2B pathway in a DCD lung EVLP model (E.J. Charles et al, unpublished 

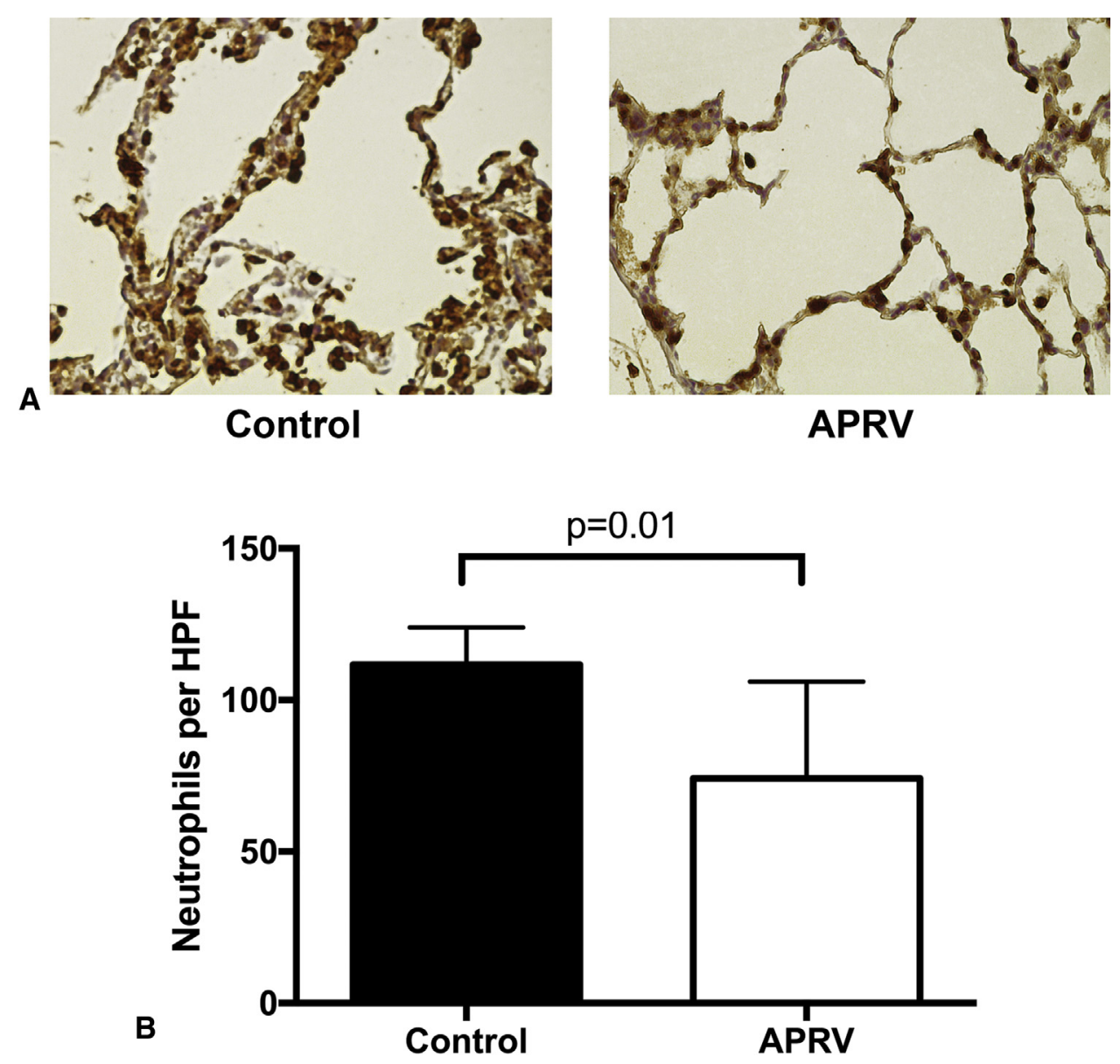

FIGURE 6. Representative images of immunohistochemistry staining for neutrophils in lung sections from both groups after transplantation and 4 hours of reperfusion (A). Neutrophil counts per high-powered field were significantly reduced in the APRV group versus control (B). APRV, Airway pressure release ventilation; $H P F$, high-powered field.

data, 2016). In the present study, IL-12 expression in the APRV group is similar to baseline post-EVLP levels and significantly less than in the control group after transplant.

Many opponents of APRV ventilation during EVLP argue that because of the absence of the chest wall, there is minimal recoil force and the lung is at high risk of barotrauma. Although this is certainly true, we found that by focusing on peak pressure $\left(\mathrm{P}_{\mathrm{High}}\right)$, we were able to maintain a lower airway pressure with better lung recruitment in the APRV mode. To confirm that we were not causing further injury, we performed histologic assessment of the lung tissue to evaluate a standard lung injury severity score previously reported. ${ }^{14,18,26}$ We demonstrate no statistical difference in lung injury scores, with a slight improvement in the APRV group compared with the controls. In addition, neutrophil activation and translocation are common phenomena after lung injury, ${ }^{27}$ and we demonstrated reduced neutrophil infiltration in the APRV group by immunohistochemistry. These results refute the theory of increased lung injury and barotrauma with APRV during EVLP.

\section{Study Limitations}

The limitations of this study include the preclinical porcine model and inherent variability among farm-raised animals. The control group and APRV group experiments were both performed by the same team of surgeons over a 3-month time period, with the controls being used previously as a baseline in another study because of the high cost of Steen. The 2-hour period of donor warm ischemia before procurement resulted in significant lung injury that was adequately rehabilitated on EVLP (mean end $\mathrm{PaO}_{2} / \mathrm{FIO}_{2}$ ratio $>300$ ) but likely would have been declined for human transplant. The EVLP protocol used in the NOVEL trial dictates a standard oxygen challenge with calculation of dynamic compliance using peak pressure, tidal volume, and PEEP in conventional mode. ${ }^{3}$ For this reason, we used a conventional mode for 5 minutes every hour for measurement of compliance. During this time, there was a massive loss of recruitment and an increase in peak pressure above previous $\mathrm{P}_{\mathrm{High}}$ despite a tidal volume setting below the volumes previously achieved with APRV. Further studies will be needed to address this issue 
before translation into human studies so that a surrogate of compliance can be calculated using $\mathrm{P}_{\mathrm{High}}$, PEEP, and corresponding tidal volume to alleviate the need for mode changes. Finally, imaging and bronchoscopy are used to aid in evaluation of the lungs during the perfusion period in the clinical setting; however, in our studies these modalities were not used.

\section{CONCLUSIONS}

A pressure-directed APRV ventilation strategy during EVLP improves the rehabilitation of severely injured DCD lungs. After transplantation, these lungs demonstrate superior lung specific oxygenation and dynamic compliance compared with lungs ventilated with standard conventional ventilation. This strategy, if implemented into current clinical EVLP protocols, could advance the field of DCD lung rehabilitation to expand the lung donor pool.

\section{Conflict of Interest Statement}

Authors have nothing to disclose with regard to commercial support.

The authors thank the University of Virginia Research Histology Core for efficient preparation of histology slides. Special thanks to Tony Herring, Cindy Dodson, and Sheila Hammond for their technical support and commitment to completion of this project.

\section{References}

1. Christie JD, Edwards LB, Kucheryavaya AY, Aurora P, Dobbels F, Kirk R, et al. The Registry of the International Society for Heart and Lung Transplantation: twenty-seventh official adult lung and heart-lung transplant report-2010. J Heart Lung Transplant. 2010;29:1104-18.

2. De Meester J, Smits JM, Persijn GG, Haverich A. Listing for lung transplantation: life expectancy and transplant effect, stratified by type of end-stage lung disease, the Eurotransplant experience. J Heart Lung Transplant. 2001;20:518-24.

3. Cypel M, Yeung JC, Hirayama S, Rubacha M, Fischer S, Anraku M, et al. Technique for prolonged normothermic ex vivo lung perfusion. J Heart Lung Transplant. 2008;27:1319-25.

4. Steen S, Liao Q, Wierup PN, Bolys R, Pierre L, Sjoberg T. Transplantation of lungs from non-heart-beating donors after functional assessment ex vivo. Ann Thorac Surg. 2003;76:244-52.

5. Yeung JC, Cypel M, Waddell TK, van Raemdonck D, Keshavjee S. Update on donor assessment, resuscitation, and acceptance criteria, including novel techniques-non-heart-beating donor lung retrieval and ex vivo donor lung perfusion. Thorac Surg Clin. 2009; 19:261-74.

6. Stock MC, Downs JB, Frolicher DA. Airway pressure release ventilation. Crit Care Med. 1987;15:462-6.

7. Henzler D. What on earth is APRV? Crit Care. 2011;15:115.

8. Daoud EG, Farag HL, Chatburn RL. Airway pressure release ventilation: what do we know? Respir Care. 2012;57:282-92.

9. Kollisch-Singule M, Emr B, Smith B, Roy S, Jain S, Satalin J, et al. Mechanical breath profile of airway pressure release ventilation: the effect on alveolar recruitment and microstrain in acute lung injury. JAMA Surg. 2014;149:1138-45.
10. Smith BJ, Lundblad LK, Kollisch-Singule M, Satalin J, Nieman G, Habashi N, et al. Predicting the response of the injured lung to the mechanical breath profile. J Appl Physiol (1985). 2015;118:932-40.

11. Terragni PP, Fanelli V, Boffini M, Filippini C, Cappello P, Ricci D, et al. Ventilatory management during normothermic ex vivo lung perfusion: effects on clinical outcomes. Transplantation. 2016;100:1128-35.

12. LaPar DJ, Laubach VE, Emaminia A, Crosby IK, Hajzus VA, Sharma AK, et al. Pretreatment strategy with adenosine A2A receptor agonist attenuates reperfusion injury in a preclinical porcine lung transplantation model. J Thorac Cardiovasc Surg. 2011;142:887-94.

13. Mulloy DP, Stone ML, Crosby IK, Lapar DJ, Sharma AK, Webb DV, et al. Ex vivo rehabilitation of non-heart-beating donor lungs in preclinical porcine model: delayed perfusion results in superior lung function. J Thorac Cardiovasc Surg. 2012;144:1208-15.

14. Wagner CE, Pope NH, Charles EJ, Huerter ME, Sharma AK, Salmon MD, et al. Ex vivo lung perfusion with adenosine $\mathrm{A} 2 \mathrm{~A}$ receptor agonist allows prolonged cold preservation of lungs donated after cardiac death. J Thorac Cardiovasc Surg. 2016;151:538-45.

15. Zhao Y, LaPar DJ, Steidle J, Emaminia A, Kron IL, Ailawadi G, et al. Adenosine signaling via the adenosine $2 \mathrm{~B}$ receptor is involved in bronchiolitis obliterans development. J Heart Lung Transplant. 2010;29:1405-14.

16. Stone ML, Sharma AK, Mas VR, Gehrau RC, Mulloy DP, Zhao Y, et al. Ex vivo perfusion with adenosine $\mathrm{A} 2 \mathrm{~A}$ receptor agonist enhances rehabilitation of murine donor lungs after circulatory death. Transplantation. 2015;99:2494-503.

17. Kollisch-Singule M, Jain S, Andrews P, Smith BJ, Hamlington-Smith KL, Roy S, et al. Effect of airway pressure release ventilation on dynamic alveolar heterogeneity. JAMA Surg. 2016;151:64-72.

18. Emaminia A, Lapar DJ, Zhao Y, Steidle JF, Harris DA, Laubach VE, et al. Adenosine A(2)A agonist improves lung function during ex vivo lung perfusion. Ann Thorac Surg. 2011;92:1840-6.

19. Tian W, Liu Y, Zhang B, Dai X, Li G, Li X, et al. Infusion of mesenchymal stem cells protects lung transplants from cold ischemia-reperfusion injury in mice. Lung. 2015;193:85-95.

20. Chang JE, Kim HJ, Yi E, Jheon S, Kim K. Reduction of ischaemia-reperfusion injury in a rat lung transplantation model by low-concentration GV1001. Eur J Cardiothorac Surg. April 26, 2016 [Epub ahead of print].

21. Lu W, Si YI, Ding J, Chen X, Zhang X, Dong Z, et al. Mesenchymal stem cells attenuate acute ischemia-reperfusion injury in a rat model. Exp Ther Med. 2015; 10:2131-7.

22. Abreu Mda M, Pazetti R, Almeida FM, Correia AT, Parra ER, Silva LP, et al. Methylene blue attenuates ischemia-reperfusion injury in lung transplantation. J Surg Res. 2014;192:635-41.

23. Farivar AS, Merry HE, Fica-Delgado MJ, McCourtie AS, MackinnonPatterson BC, Mulligan MS. Interleukin-6 regulation of direct lung ischemia reperfusion injury. Ann Thorac Surg. 2006;82:472-8.

24. Teng MW, Bowman EP, McElwee JJ, Smyth MJ, Casanova JL, Cooper AM, et al. IL-12 and IL-23 cytokines: from discovery to targeted therapies for immunemediated inflammatory diseases. Nat Med. 2015;21:719-29.

25. Cero FT, Hillestad V, Loberg EM, Christensen G, Larsen KO, Skjonsberg OH. IL-18 and IL-12 synergy induces matrix degrading enzymes in the lung. Exp Lung Res. 2012;38:406-19.

26. Reece TB, Ellman PI, Maxey TS, Crosby IK, Warren PS, Chong TW, et al. Adenosine A2A receptor activation reduces inflammation and preserves pulmonary function in an in vivo model of lung transplantation. J Thorac Cardiovasc Surg. 2005;129:1137-43.

27. Laan M, Cui ZH, Hoshino H, Lotvall J, Sjostrand M, Gruenert DC, et al. Neutrophil recruitment by human $\mathrm{IL}-17$ via $\mathrm{C}-\mathrm{X}-\mathrm{C}$ chemokine release in the airways. J Immunol. 1999;162:2347-52.

Key Words: lung transplant, EVLP, ex vivo lung perfusion, APRV, airway pressure release ventilation, lung injury, ischemia reperfusion 